\title{
A simple disc technique for the presumptive identification of Legionella pneumophila
}

\author{
MG SMITH \\ From the Department of Pathology, Kingston Hospital, Galsworthy Road, Kingston-upon-Thames, Surrey
}

SUMMARY A method is described for the presumptive identification of Legionella pneumophila by the formation of satellite colonies around filter paper discs impregnated with ferric pyrophosphate and L-cysteine hydrochloride on a deficient basal medium. This technique simplifies the differentiation of picked colonies of $L$ pneumophila from other organisms in mixed cultures from environmental and contaminated clinical samples.

Several culture media have been developed for the cultivation of $L$ pneumophila and with the addition of antibiotics to produce semiselective media, the isolation of $L$ pneumophila from mixed cultures has been facilitated. ${ }^{1-4}$ Until now $L$ pneumophila has been initially identified by colonial appearance, morphology when stained and failure to grow on non-specialised media such as blood agar. ${ }^{5} \mathrm{~A}$ method has now been devised which simplifies the identification of isolates when dealing with large numbers of suspect colonies from mixed cultures.

\section{Material and methods}

The basal medium for this identification technique is a modified charcoal yeast extract medium (BCYE $\alpha$ base) without ferric pyrophosphate and L-cysteine hydrochloride. ${ }^{4}$ Filter paper discs impregnated with ferric pyrophosphate and L-cysteine hydrochloride provide the source of the Legionella growth supplements.

BCYI $\alpha-B A S E$

This consisted of $10 \mathrm{~g}$ yeast extract (Difco), $1.5 \mathrm{~g}$ Norit A activated charcoal (Sigma) $10 \mathrm{~g}$ ACES (Sigma) $1 \mathrm{~g} \alpha$-ketoglutarate-monopotassium salt (Sigma), $12 \mathrm{~g}$ Agar No 3 (Oxoid) distilled water to one litre, adjusted to $\mathrm{pH} 6.9$ with $8 M$ potassium hydroxide and autoclaved at $121^{\circ} \mathrm{C}$ for $15 \mathrm{~min}$. Culture plates were dried before use.

FILTER PAPER DISCS

Discs ( $5 \mathrm{~mm}$ diam) were punched from Postlip filter paper sheets (Evans, Adlard \& Co Ltd,
Winchcombe, UK) and decontaminated in a hot air oven at $65^{\circ} \mathrm{C}$ for $24 \mathrm{~h}$.

\section{GROWTH SUPPLEMENTS}

A filter sterilised stock solution of ferric pyrophosphate $12.5 \mathrm{mg} / \mathrm{ml}$, L-cysteine hydrochloride $20 \mathrm{mg} / \mathrm{ml}$ was prepared. The sterile discs were saturated with $10 \mu$ l of stock solution each, dried at $37^{\circ} \mathrm{C}$ and stored with silica gel at $4^{\circ} \mathrm{C}$ for up to three months.

BCYE $\alpha$-base was inoculated by streaking individual suspect colonies across the culture plate using sterile cotton applicator swabs, each streak being at least $70 \mathrm{~mm}$ in length. Standard $90 \mathrm{~mm}$ Petri dishes will accommodate five streaks and $100 \mathrm{~mm}$ square dishes will accommodate 10 streaks. The impregnated discs were placed in the centre of each streak and the plates incubated for up to three days at $35^{\circ} \mathrm{C}-37^{\circ} \mathrm{C}$ in a humidified incubator. A known $L$ pneumophila culture was included on each plate as a control.

\section{Results}

See Fig. 1. Typical satellite growth was produced around the discs by stock cultures of $L$ pneumophila serogroups 1 to 6 , eight wild isolates of serogroup 1 , one wild isolate of serogroup 4 and one wild isolate ungrouped.

All non-Legionella organisms examined grew across the streaks and did not produce satellite growth. These organisms included Haemophilus influenzae from a clinical sample as well as Pseudomonas spp, Flavobacterium spp and other unidentified water organisms from 56 environmental samples. 


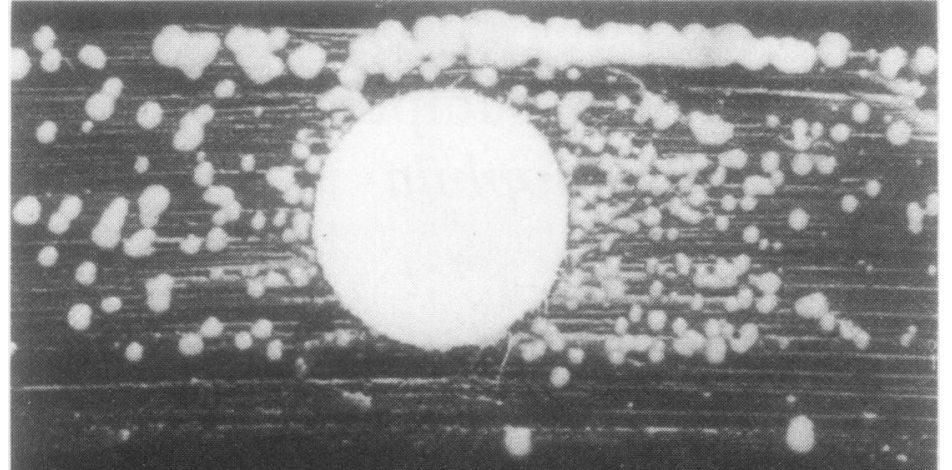

Fig. 1 Upper disc with satellite colonies of $L$ pneumophila; lower disc with nonLegionella organism (in this case Haemophilus influenzae) failing to produce satellite colonies.

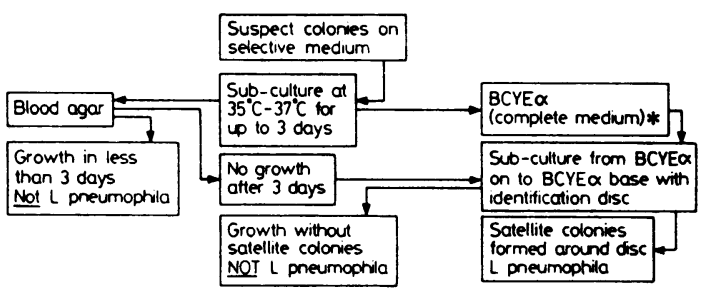

* BCYEa base. $0 \mathrm{Lg} /$ litre $\mathrm{L}$-cysteine hydroctloride and $025 \mathrm{~g} /$ litre terric pyrophosphate

Fig. 2 Flow pattern for the use of the disc technique.

\section{Discussion}

When this laboratory investigated a recent case of hospital-acquired Legionnaires' disease, large numbers of environmental samples were processed using semiselective media. These samples on primary isolation yielded mixed cultures of $L$ pneumophila and environmental organisms. Many of these environmental organisms resembled $L$ pneumophila colonially and grew slowly on blood agar. At first, time-consuming immunofluorescent serogrouping was employed to identify isolates. However, using the flow pattern shown in Fig. 2, the disc technique described was found to be much simpler when dealing with the large numbers of potential Legionella isolates encountered.

Most of the $L$ pneumophila cultures tested produced satellite growth in 18-24 h with a few requiring $24-72 \mathrm{~h}$, this variation was due to inoculum size. All the non-Legionella organisms which grew slowly on blood agar (more than three days), grew well on BCYE $\alpha$-base in less than three days and growth was not improved by the impregnated discs so they were easily differentiated from $L$ pneumophila.

The BCYE $\alpha$-base and the impregnated discs were simple to make from the ingredients already used in current Legionella culture media. It was found that Oxoid Legionella growth supplement (SR94) could be used as a source of ferric pyrophosphate and L-cysteine hydrochloride.

I wish to thank Dr SP Fisher-Hoch and Dr JO'H Tobin for their advice and encouragement. I also wish to thank Ms M Stone for secretarial assistance in the preparation of this manuscript. 


\section{References}

' Feely JC, Gorman GW, Weaver RE, Mackel DC, Smith HW. Primary isolation media for Legionnaires disease bacterium. $J$ Clin Microbiol 1978;8:320-5.

' Feely JC, Gibson RJ, Gorman GW, et al. Charcoal yeast extract agar: primary isolation medium for Legionella pneumophila. $J$ Clin Microbiol 1979;10:437-41.

${ }^{3}$ Dennis PJ, Taylor JA, Barrow GI. Phosphate buffered, low sodium chloride blood agar medium for Legionella pneumophila (correspondence). Lancet 1981;ii:636.
+ Edelstein PH. Improved semiselective medium for isolation of Legionella pneumophila from contaminated and environmental specimens. J Clin Microbiol 1981;14:298-303.

${ }^{5}$ McDade JE, Shepard CC, Fraser DW. Legionnaires' disease. Isolation of a bacterium and demonstration of its role in other respiratory disease. N Engl J Med 1977;297:1197-203.

Requests for reprints to: Mr MG Smith, Department of Microbiology, Kingston and Esher Health Authority, 37 Coombe Road, Kingston upon Thames, Surrey KT2 7BD, England. 